\title{
Synthesis of ordered mesoporous silica MCM-41 with controlled morphology for potential application in controlled drug delivery systems
}

\author{
(Síntese de sílica mesoporosa ordenada tipo MCM-41 \\ com controle morfológico para potencial aplicação em sistemas \\ de liberação controlada de fármacos)
}

\author{
D. M. Oliveira ${ }^{1}$, A. S. Andrada ${ }^{1 *}$ \\ ${ }^{1}$ Universidade Federal de Itajubá, R. Irmã Ivone Drumond 200, 35903-087, Itabira, MG, Brazil
}

\begin{abstract}
MCM-41 is one of the most studied mesoporous ceramics for drug delivery systems. Its high specific surface area and mesoporosity allow high adsorption capacity. Even though there are many studies published in the biomedical field, there are no reports of commercial applications of MCM-41 so far. One of the possible justifications is the lack of morphological control during conventional synthesis. Therefore, modifications in the reaction parameters of the MCM-41 conventional synthesis were tested in this paper, aiming to obtain particles with reduced diameter and agglomeration. It was observed that both the increase in the water molar proportion and the decrease in the stirring time resulted in particles with reduced size. Furthermore, the control of the tetraethyl orthosilicate (TEOS) dropping rate and the addition of triethylamine (TEA) improved the dispersion of the system, but they also decreased the particle size, and therefore van der Waals interactions promoted re-agglomeration.

Keywords: MCM-41, controlled drug release, reduction of particle size, increase in dispersion.
\end{abstract}

Resumo

O MCM-41 é uma das cerâmicas mesoporosas mais estudadas para sistemas de liberação controlada de fármacos. Sua elevada área superficial específica e mesoporosidade permitem alta capacidade de adsorção. Apesar dos inúmeros trabalhos publicados na área biomédica, até o presente momento não há relatos de aplicações comerciais do MCM-41. Uma possível justificativa é a falta de controle morfológico durante a síntese convencional. Portanto, modificações nos parâmetros reacionais da síntese convencional do MCM-41 foram testadas neste artigo com o objetivo de obter partículas com diâmetro e estado de aglomeração reduzidos. Foi observado que o aumento na proporção molar de água e a diminuição no tempo de agitação resultaram em partículas com tamanho reduzido. Além disso, o controle do gotejamento do tetraetilortossilicato (TEOS) e a adição da trietilamina (TEA) melhoraram a dispersão do sistema, porém eles também reduziram o tamanho das partículas e, assim, interações do tipo van der Waals promoveram a reaglomeração.

Palavras-chave: MCM-41, liberação controlada de fármacos, redução de tamanho de partículas, aumento da dispersão.

\section{INTRODUCTION}

Ordered mesoporous ceramics are characterized by the presence of ordered porosity, with a diameter between 2 and $50 \mathrm{~nm}$, but disordered silica walls [1]. Those materials were first developed in the 1990's aiming to increase the diameter of zeolites pores and enable its application in adsorption and catalysis. In 1992, scientists from Mobil Oil Co. synthesized ordered mesoporous materials of the M41S type, family to which MCM-41 belongs using surfactants to promote the formation of porous structures. Instead of

*andrezaandra@unifei.edu.br

(D) https://orcid.org/0000-0002-6884-8345 using small organic molecules as templating compounds, as in the case of zeolites, long chain surfactant molecules were employed as the structure-directing agent during the synthesis of these highly ordered materials [1]. Currently, there are many families of mesoporous materials, which are produced by distinct synthesis methods and reactants. The most frequently reported families are MCM-n (Mobil Corporation Matter), SBA-n (Santa Barbara), MSU-n (Michigan State University), KIT-n (Korean Institute of Technology), FSM-n (folded sheet materials), FDU (Fudan University) and AMS-n (anionic mesoporous silica) [1].

Despite starting in the catalysis industry, mesoporous materials, especially MCM-41, have been explored for applications in the biomedical area, as scaffolds for tissue 
engineering, in gene therapy, anti-cancer therapy, and drug delivery systems. Those applications are possible because of the unique properties of the mesoporous ceramics, which include high specific surface area and pore volume, biocompatibility in vitro and in vivo, bioactivity, thermal and chemical stability, tunable particle and pore size, and the possibility of functionalization [1, 2]. Vallet-Regi and coworkers [3] were pioneers in the studies of MCM41 as potential materials for controlled drug delivery. The interest in drug delivery systems is justified by the necessity of improving the therapeutic response of conventional medicines. Advantages of drug delivery systems over traditional systems include the ability to deliver drugs to a specific site, the small variability in systemic drug concentrations and the reduction in toxic metabolites. Nowadays, MCM-41 is one of the most studied mesoporous ceramics to be employed as a matrix for controlled drug release. Particularly, its high specific surface area provides the possibility to load a high amount of drugs on its surface [4], leading to a more efficient system. In addition, its mesoporosity allows the encapsulation of molecules or mixture of molecules with variable diameter, shape, and functionality. Even though there are many scientific projects being developed in the field of controlled drug delivery, commercial applications of MCM-41 were not reported so far. One of the possible justifications is the lack of morphological control when using conventional methods to synthesize mesoporous ceramics; particles with various size and shape are obtained. Consequently, the properties of mass transfer at the nanometric level are affected, and they are fundamental to the controlled release and delivery of drugs in biological systems [5].

The size of the mesoporous silica particles has a fundamental role in the absorption and capture of the material by the cells, in the penetration through tissues, in the accumulation of particles in non-target organs, in the time for circulation in the bloodstream, and in the release rate of the drug adsorbed on the external or internal surface of the pores $[2,6]$. Therefore, the control of the particle size during the synthesis is essential to obtain efficient drug delivery systems. For biomedical applications, it is desired to obtain uniform nanoparticles with 50 to $300 \mathrm{~nm}$ in diameter since larger particles may not be able to cross physical membranes in the body and smaller particles are harder to synthesize keeping their mesoporosity [7]. Another factor that needs to be controlled during the synthesis of ordered mesoporous materials is the agglomeration of particles. When the size is reduced, Brownian motion and van der Waals forces act more intensely, increasing the contact and interaction between particles and therefore leading to agglomeration. In the biomedical area, preventing this effect is crucial to control the physical-chemical properties of the nanoparticles, such as particle size distribution, surface/volume ratio, and reactivity; those properties can increase the toxicity and the probability of blocking vascular and lymphatic channels in the organism $[8,9]$.

To obtain mesoporous silica materials, one of the most used technique is the surfactant-assisted sol-gel. The surfactant acts as a structure directing agent, and the hydrolysis and condensation reactions occur on the surface of the micelles [10]. Modifications on the synthesis parameters, such as $\mathrm{pH}$, reaction temperature, type and concentration of catalyst, and molar ratios between reactants, have been explored in order to reduce the particle diameter and to control its agglomeration $[6,11,12]$. For example, in [11] mesoporous silica nanoparticles were obtained from tens to hundreds of nanometers in diameter, through the variation in water/methanol ratio. In [12], in turn, nanoparticles of mesoporous silica with diameters between 65 and 740 $\mathrm{nm}$ were obtained by altering the TEOS/surfactant ratio under dilute conditions. The effect of the base catalyst $(\mathrm{NaOH})$ concentration on the diameter of mesoporous silica nanoparticles was evaluated in [6]. It was shown that the decrease in the $\mathrm{NaOH}$ concentration from 22.5 to $12.5 \mathrm{mM}$ led to smaller MCM-41 particles, with a diameter of $90 \mathrm{~nm}$. However, the connectivity between the primary particles increased leading to large agglomerates in the suspension. In addition, in [13] the effect of different functional groups for the surface-modification of silica nanoparticles was studied. The samples were prepared using a water-in-oil microemulsion; functional groups including carboxylate, amine, amine/phosphonate, poly(ethylene glycol), octadecyl, and carboxylate/octadecyl were incorporated to the surface of the nanoparticles. Characterization techniques such as scanning electron microscopy (SEM), dynamic light scattering (DLS), and zeta potential analysis proved that addition of inert functional groups to the surface of the amine-modified silica nanoparticles could reduce the agglomeration among them. The authors [13] observed that a highly negative zeta potential was obtained when the proper proportion of inert functional groups (e.g., methylphosphonate) to active functional groups (e.g., amino groups) was added to the surface of silica nanoparticles, which enhanced the dispersion of the system and the chemical stability of biomolecules. Thus, modifications of the reaction parameters of the conventional synthesis of MCM-41 mesoporous silica were made in this study in order to obtain particles with size and dispersion conditions suitable to possible applications in drug delivery systems. For this, the stirring time and the water to tetraethyl orthosilicate (TEOS) molar ratio were altered. Moreover, it was analyzed the influence of adding TEOS to the solution in a controlled manner and the addition of triethylamine (TEA) dispersion agent to control the nucleation process and to inhibit the agglomeration of particles.

\section{MATERIALS AND METHODS}

Materials: the reactants used in the synthesis of the mesoporous silica were: tetraethyl orthosilicate (TEOS) 98\%, Sigma Aldrich, as a source of silica; hexadecyltrimethylammonium bromide (CTAB) $\geq 96 \%$, Fluka, as a structure directing agent; tetramethylammonium hydroxide (TMAOH 25\% in water) as the catalyst of the sol-gel reaction; 
and triethylamine (TEA) as a dispersion agent. The TEA is able to inhibit the growth of the mesoporous silica particles and, at the same time, acts as a base catalyst [2].

Procedures: the methodology used in this work was based on the procedure of synthesis of MCM-41 mesoporous silica proposed in [3] with some modifications aiming to reduce the size and agglomeration of the particles. Table I shows the reaction conditions for the synthesis of the mesoporous samples. In this table, the identification of the samples, as well as the reactants, temperature and stirring time for each synthesis, can be found. For the synthesis of the conventional mesoporous silica (MCM CV), solution A was obtained by dissolving the surfactant $\mathrm{CTAB}$ in distilled water. Magnetic stirring was kept constant (approximately $300 \mathrm{rpm}$ ) until the solution became transparent. In the meantime, solution B was prepared by adding $10 \%$ of the total volume of TEOS in TMAOH. This mixture was homogenized and then poured into solution $\mathrm{A}$, increasing the magnetic stirring to approximately $500 \mathrm{rpm}$. After $30 \mathrm{~min}$ of homogenization, the remaining TEOS was added dropwise to the solution. The reaction was maintained under magnetic stirring at room temperature for $12 \mathrm{~h}$. Next, the container with the silica solution was sealed and taken into an oven for the hydrothermal treatment at $80{ }^{\circ} \mathrm{C}$ for $24 \mathrm{~h}$. This sample was prepared with a molar proportion of 1.000/0.114/21.741/0.301/0.000 $\mathrm{TEOS} / \mathrm{CTAB} / \mathrm{H}_{2} \mathrm{O} / \mathrm{N}\left(\mathrm{CH}_{3}\right)_{4} \mathrm{OH} / \mathrm{TEA}$. The mixture was filtered under vacuum and washed with water and ethanol $(5: 1 \mathrm{v} / \mathrm{v})$. This powder was then dried in an oven at $60{ }^{\circ} \mathrm{C}$ for $24 \mathrm{~h}$ and calcined in order to remove the template. The conditions for calcination were a heating rate of $1{ }^{\circ} \mathrm{C} / \mathrm{min}$ from room temperature to $600{ }^{\circ} \mathrm{C}$ and cooling in air. The same procedure was followed for all synthesized mesoporous particles, but with some modifications, according to Table I. The synthesis temperature was kept constant for all samples $\left(25^{\circ} \mathrm{C}\right)$. The stirring time, however, was reduced from 12 to $2 \mathrm{~h}$ for all samples with TR on their denomination (MCM CV TR, MCM CV TR DL, MCM CV TR DL ${ }_{\text {CG }}$ MCM CV TR DL TEA ${ }_{\mathrm{CG}}$ ). The synthesis of the sample MCM CV TR DL ${ }_{\text {CG }}$ was performed with reduced stirring time, in a diluted medium and with controlled addition of TEOS. Furthermore, another synthesis was carried out following the same parameters adopted for the sample MCM CV TR
$\mathrm{DL}_{\mathrm{CG}}$ but adding the triethylamine reactant to the mixture of surfactant and water (solution A); this sample was called MCM CV TR DL TEA ${ }_{\text {CG }}$.

Since the formation of particles of mesoporous silica is resultant from nucleation and growth mechanisms and those processes are time-related, the stirring time (or homogenization time) was reduced to obtain particles with smaller diameters. The TEOS monomers obtained during hydrolysis connect and form clusters, which are unstable until they reach a critical diameter. The growth of those particles happens due to hydrolyzed monomers being constantly added to their surface [14] and the diameter of the clusters per unit volume is directly proportional to the reaction time. Several methodologies for obtaining mesoporous silica are reported in the literature with stirring time between 1 and $24 \mathrm{~h}[5,7$, $15,16]$. Therefore, it was considered that the shortening of the stirring time with respect to the conventional synthesis was a valid proposal. The increase in the molar proportion of water in the reactant medium promotes the increase in the TEOS hydrolysis rate during the reaction, which happens due to the expansion of the number of active sites for the formation of silica nuclei. Therefore, smaller particles can be obtained. The source of silica (TEOS) added in a controlled way also affects the morphology of the particles. It acts in the stages of nucleation and growth and directly affects the concentration of hydrolyzed silica units in the reaction medium, avoiding then the nucleation of the second group of nuclei with different diameters as well as promoting a better dispersion of the system $[17,18]$. Finally, TEA can be used as a dispersion agent, co-inhibitor of the particle growth and catalyst of the sol-gel reaction, resulting in mesoporous silica nanoparticles well dispersed in the reaction medium [2]. The sample MCM CV was the reference for the discussion of results since it was obtained with the same synthesis conditions suggested in [3] for mesoporous silica MCM-41.

Characterization techniques: nitrogen adsorption isotherms of samples were obtained at $77 \mathrm{~K}$ using a Quantachrome Nova 2200 adsorption analyzer. Before the adsorption measurements, the samples were outgassed for $24 \mathrm{~h}$ at $473 \mathrm{~K}$. All data analyses were performed using the NovaWin V.10 1997-2007 Quantachrome Instr. software (Boynton Beach, USA). SEM characterization was

Table I - Reaction conditions for the synthesis of mesoporous materials.

[Tabela I - Condições reacionais para a sintese dos materiais mesoporosos.]

\begin{tabular}{|c|c|c|}
\hline Sample identification & $\begin{array}{c}\text { Molar proportion } \\
\text { TEOS/CTAB/ } \mathrm{H}_{2} \mathrm{O} / \mathrm{N}\left(\mathrm{CH}_{3}\right)_{4} \mathrm{OH} / \mathrm{TEA}\end{array}$ & Stirring time $(\mathrm{h})$ \\
\hline $\mathrm{MCM} \mathrm{CV}^{1}$ & $1.000 / 0.114 / 21.741 / 0.301 / 0.000$ & 12 \\
\hline MCM CV TR 2 & $1.000 / 0.114 / 21.741 / 0.301 / 0.000$ & 2 \\
\hline MCM CV TR DL ${ }^{3}$ & $1.000 / 0.114 / 2174.100 / 0.301 / 0.000$ & 2 \\
\hline MCM CV TR DL ${ }_{\mathrm{CG}}^{4}$ & $1.000 / 0.114 / 2174.100 / 0.301 / 0.000$ & 2 \\
\hline MCM CV TR DL TEA $_{\mathrm{CG}}{ }^{5}$ & $1.000 / 0.114 / 2174.100 / 0.301 / 0.090$ & 2 \\
\hline
\end{tabular}


performed in a Tescan Vega Easy Probe microscope operating at $15 \mathrm{kV}$. The images were obtained with magnifications of 10000 and 50000 times. To obtain the images, the powder samples were dispersed into acetone using an ultrasonic bath and then pipetted on an aluminum sample holder. The samples were exposed to a cloud of sublimated gold for 10 $\mathrm{s}$, enough time for them to be covered by a thin metal layer with approximately $2.5 \mathrm{~nm}$ thickness. Calculations: BETspecific surface area, $\mathrm{S}_{\mathrm{BET}}$, was calculated from adsorption data in the relative pressure interval of 0.05 to $0.25 \mathrm{P} / \mathrm{P}_{0}$ [19]. A cross-sectional area of $0.162 \mathrm{~nm}^{2}$ was used for the nitrogen molecule in the BET calculations. The total pore volume, $\mathrm{V}_{\mathrm{p}}$, was calculated from the amount of $\mathrm{N}_{2}$ adsorbed at the highest $\mathrm{P} / \mathrm{P}_{0}(0.99)$ [20]. The mesopore diameter was estimated from the position of the peak of the mesopore size distribution curves. Even though there are several methods to evaluate the mesoporous size distribution, in this work it was chosen the Barrett-Joyner-Halenda (BJH) method, which presents a good approximation for the real pore distribution and is a commonly used method to analyze the pore size distribution in the mesopore scale [21]. For the calculation of mesopore size by means of the BJH method, the data from the desorption curve was used, and the mesopore volume was determined in the adsorption-desorption point.

\section{RESULTS AND DISCUSSION}

\section{Nitrogen adsorption}

The nitrogen adsorption isotherm for the reference sample (MCM CV) is presented in Fig. 1. It is possible to observe that this sample exhibited an isotherm type IV, typical of mesoporous materials, according to the IUPAC classification [20]. Five well-defined regions can be seen in this isotherm, which are described in [22, 23]. Initially, in I there was adsorption in low relative pressure regions $\left(\mathrm{P} / \mathrm{P}_{0}\right.$ : $10^{-5}$ to 0.02 ) due to the filling of the micropores. In region II, a linear increase of the adsorbed volume happened due to the monolayer and multilayer adsorption on the surface of the material, including the surface of the mesoporous. In region III, the increase of the adsorbed volume at intermediate relative pressures (appearance of an inflection point between 0.25 and $0.50 \mathrm{P} / \mathrm{P}_{0}$ ) was attributed to the capillary condensation phenomenon in the mesopores. In this point, the amount of adsorbed gas increased sharply with a small change in pressure due to the condensation of the adsorbate molecules below their vapor pressure. The adsorbate in the liquid state filled up the primary mesopores until the point in which the slope of the curve decreased, almost forming plateau. In region IV, the increase in the adsorbed volume under high relative pressures was associated with the multilayer adsorption and/or to the condensation of the liquid in the secondary mesopores. Finally, in V, the increase of the adsorbed volume corresponded to the filling of the voids between the particles that can be considered as porosity (yellow region in Fig. 1).

This isotherm is reversible with a hysteresis loop. This hysteresis loop can be attributed to the capillary

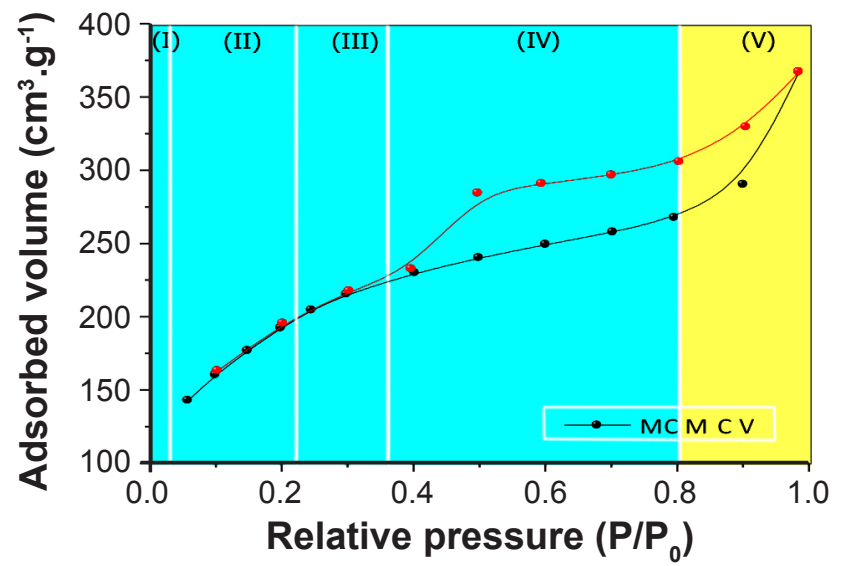

Figure 1: $\mathrm{N}_{2}$ adsorption isotherm for the mesoporous silica MCM $\mathrm{CV}$. The black line represents adsorption and the red one desorption. [Figura 1: Isoterma de adsorção de $\mathrm{N}_{2}$ da sílica mesoporosa MCM CV.A curva preta representa a adsorção e a vermelha a dessorção.]

condensation and it occurs because of the delay in pore condensation. Fig. 2 shows the differentiation of the six types of hysteresis according to the shape of the adsorption isotherm, as described by IUPAC [24]. The type H1 hysteresis loop can be found in materials with pores of regular cylindrical and/or polyhedral shape with open ends; they are typical of mesoporous materials such as SBA-15 [24]. This type of hysteresis is very symmetrical and the adsorption and desorption branches are very similar. They remain parallel as the relative pressure increases and exhibit asymptotic behavior in the meeting regions. In other words, they get closer together as the loop reaches its lower limit. The type $\mathrm{H} 2$ hysteresis is formed when the material exhibits cylindrical pores, open and closed with necking, resulting in an irregular morphology with a bottle aspect. In this type of hysteresis, one of the curves is steeper than the other one as a consequence of the pore interconnectivity [25]. Furthermore, the slope of the desorption curve is related to the pore-blocking effect inside the neck, and the diameter of the resulting neck defines two distinct types of $\mathrm{H} 2$ hysteresis: (a) and (b) types. In the H2(a) type, the diameter of the neck is narrower than in the type H2(b) [24]. In the hysteresis loop H3, the pores have a wedge or slit geometry, resulting from agglomerates of parallel plates-shaped particles [24]. $\mathrm{H} 4$ type is related to pores with the same geometry as the H3 type, but with smaller diameters [26]. H5 type hysteresis loop is not very common, but it can be attributed to materials with open and partially open mesopores [24]. It is possible to observe in Fig. 2 that in the H3, H4 and H5 hysteresis types the desorption branch tends to be perpendicular to the adsorption branch in the closure region, at lower relative pressures.

Comparing Figs. 1 and 2, it is possible to identify a hysteresis loop with $\mathrm{H} 4$ type in the sample MCM CV since the adsorption and desorption curves were more horizontal; this behavior is typical of complex materials with both micropores and mesopores [27, 28]. It is worth mentioning that, as described in the region $\mathrm{V}$ of the isotherm shown in Fig. 1, the abrupt increase in the adsorbed volume in regions 


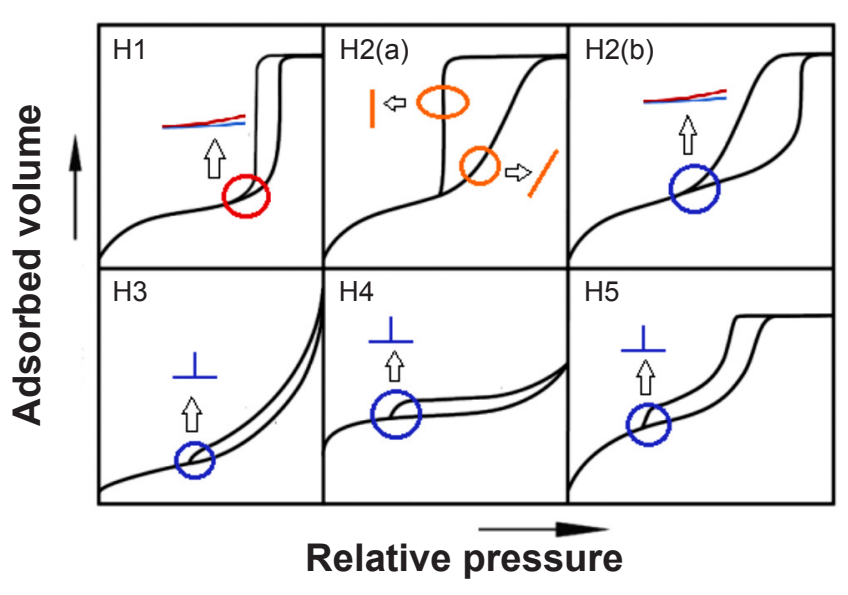

Figure 2: Different types of hysteresis loops according to the shape of the curves (adapted from [24]).

[Figura 2: Diferenciação dos tipos de loop de histerese pela forma das curvas (adaptado de [24]).]

of elevated relative pressure is characteristic of pores formed between the particle agglomerates (macroporosity). In the paragraphs below, it is presented a comparative analysis of the isotherm profiles for all the synthesized samples, as well as their textural parameters (shown in Table II), which were obtained from the nitrogen adsorption measurements.

Effect of the reduction in the stirring time: comparing the adsorption isotherms for the mesoporous samples MCM CV and MCM CV TR (Figs. 3a and 3b, respectively) synthesized with the same reactants but different stirring time (12 and $2 \mathrm{~h}$, respectively), it was observed that both samples presented type IV isotherm and type H4 hysteresis loop. Another aspect observed in these adsorption isotherms was the abrupt increase in the volume of adsorbed gas in the interval of high relative pressures (after the capillary condensation region); it can be attributed to the multilayer adsorption on the external surface of the material [29]. The isotherm tends to a zero slope after the pores are totally filled with the adsorbate, and this slope reflects the small external surface area and/or the insignificant number of secondary particles, i.e. agglomerates [30]. Therefore, the steep slope of the curve in the region of high relative pressure for MCM CV TR sample indicated the presence of macroporosity, resultant from the irregular shape of the particles and/or formation of agglomerates. It is known that the reaction time has a direct correlation to the processes of the nucleation and growth of particles [31]. As the reaction time increases, the nuclei formed during the nucleation stage start to grow. The rate of particle growth depends on the diffusion of atoms from inside the bulk towards the surface and on the incorporation of those atoms to the surface [32]. Each one of those mechanisms leads to a different correlation between particle size and reaction time but, in both cases, there is an increase in particle diameter. In addition, in the late stages of particle growth, the larger particles grow in the expense of the smaller particles. This mechanism is known as Ostwald ripening and the particles increase in size but decrease in number [32]. Therefore, it was expected particles with a smaller diameter for the synthesis with the reduced reaction (stirring) time. However, according to the Table II, the specific surface area was higher for MCM CV than MCM CV TR, possibly indicating smaller particle sizes for the former sample; this behavior was the opposite from the predicted. The specific surface areas for the samples MCM CV and MCM CV TR were, respectively, 698 and $642 \mathrm{~m}^{2} \cdot \mathrm{g}^{-1}$. A possible explanation for those numbers is that the reduction in the stirring time resulted in the formation of particles with reduced diameter, however, the intensity of the van der Waals interactions between those small particles increased, leading to an agglomerated system. Thus, an increase in the agglomeration of the particles in the MCM CV TR sample is believed to be the explanation for a smaller specific surface area. Besides, the microscopy images for the samples under discussion (section below) show that both of them were agglomerated, but MCM CV TR apparently presented smaller particles. According to Table II, the diameter of the mesopores was not altered with the reduction of the homogenization time; it was approximately $3.6 \mathrm{~nm}$ for both samples. This was an expected result since the pore size is usually changed by aging temperature [33] and by using different surfactants and/or pore expander agent $[34,35]$. Moreover, the mesopore volume increased by about $19 \%$ for the MCM CV TR sample.

Effect of the increase in the molar proportion of water: the adsorption profile for the samples MCM CV TR and MCM CV TR DL are presented in Figs. $3 b$ and 3c, respectively. It can be observed that both samples had a type IV adsorption isotherm, according to the IUPAC classification. However, the width of the hysteresis loop decreased significantly with the increase of the water amount in the reaction medium. The adsorption phenomenon was studied in MCM-41 silicas with variable pore sizes [36]. It was reported that the changes in the pore size, as well as the changes in the

Table II - BET and BJH parameters for the mesoporous silica samples.

[Tabela II - Parâmetros de BET e BJH para as amostras de sílica mesoporosa.]

\begin{tabular}{ccccc}
\hline Sample & $\mathrm{S}_{\mathrm{BET}}\left(\mathrm{m}^{2} \cdot \mathrm{g}^{-1}\right)$ & $\mathrm{R}$ & $\mathrm{D}_{\mathrm{P}}(\AA)$ & $\mathrm{V}_{\mathrm{P}}\left(\mathrm{cm}^{3} / \mathrm{g}\right)$ \\
\hline MCM CV & 698.00 & 0.99994 & 36.12 & 0.57 \\
MCM CV TR & 642.30 & 0.99975 & 35.82 & 0.68 \\
MCM CV TR DL & 970.77 & 0.99860 & 35.94 & 0.68 \\
MCM CV TR DL $_{\mathrm{CG}}$ & 739.02 & 0.99967 & 36.63 & 0.62 \\
MCM CV TR DL TEA $_{\mathrm{CG}}$ & 919.31 & 0.99998 & 35.78 & 1.29 \\
\hline
\end{tabular}



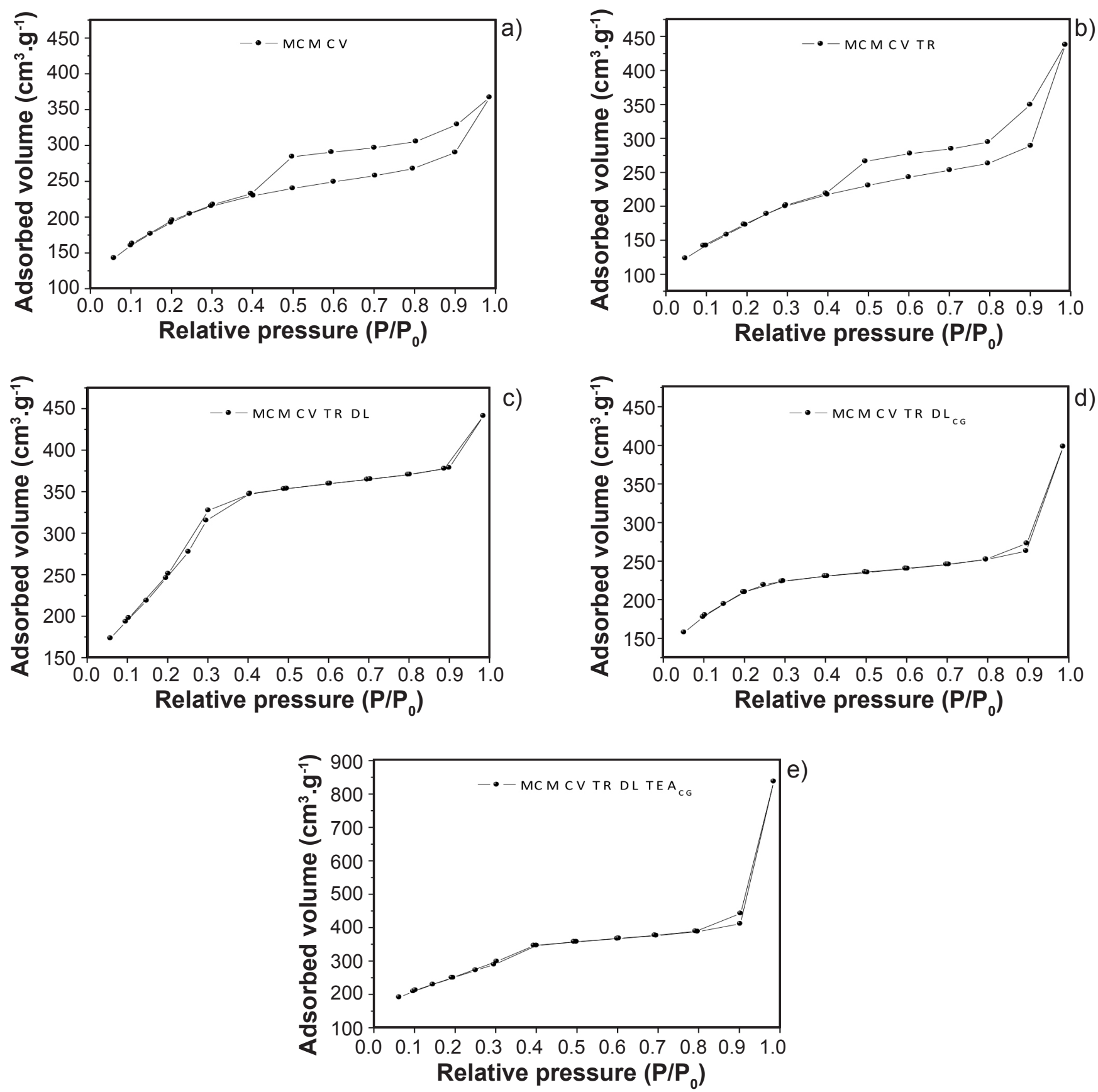

Figure 3: $\mathrm{N}_{2}$ adsorption isotherm for the samples: a) MCM CV; b) MCM CV TR; c) MCM CV TR DL; d) MCM CV TR DL ; and e) MCM CV TR DL TEA ${ }_{\mathrm{CG}}$. Note that in (e) the $\mathrm{y}$-axis scale range is larger than in the other graphs.

[Figura 3: Isotermas de adsorção de $N_{2}$ das amostras: a) MCM CV; b) MCM CV TR; c) MCM CV TR DL; d) MCM CVTR DL CV TR DL TEA $A_{C G}$. Note que em (e) o intervalo da escala do eixo y é maior do que nos outros gráficos.]

pore size distribution in the mesoporous samples, resulted in changes in the width of the hysteresis loop; a non-uniform pore size distribution led to a large hysteresis loop, while a reduced pore size caused a narrowing of the hysteresis loop. The specific surface area was significantly increased when comparing the samples MCM CV TR and MCM CV TR DL, as one can see in Table II (from 642 to $970 \mathrm{~m}^{2} . \mathrm{g}^{-1}$ ), possibly indicating a decrease in particle size and/or an improvement on the dispersion of the particles. Besides, analyzing the SEM images in next section for both samples, it was noticed that the particles in the sample MCM CV TR DL were smaller and less agglomerated than the sample MCM CV TR. The reduction on the particle size with the increase in the molar ratio of water/TEOS is likely to be a result of the expansion in the number of reactive sites and hydroxyl groups for the silica centers, accelerating the hydrolysis reaction [37]. It is reported in the literature that the formation of mesoporous silica particles is a consequence of the nucleation and growth stages. The nucleation happens after the saturation limit during the hydrolysis, in which there are reactive silica 
nuclei, and the growth happens through the condensation of monomeric silicic acid $\left[\mathrm{Si}\left(\mathrm{OH}_{4}\right)\right]$ on the surface of the clusters [38]. Considering that the excess of water led to more functional groups for the formation of nuclei, a larger number of silica centers was formed during the nucleation. As a result, smaller particles were obtained. According to Table II, there was no significant change in the mesopore size after increasing the molar proportion of water during the synthesis (approximately $3.6 \mathrm{~nm}$ for both MCM CV TR and MCM CV TR DL samples). One possible reason for the narrowing on the hysteresis loop in Fig. $3 \mathrm{c}$ is a more homogeneous and uniform pore size distribution [36]. In addition, the effect of the dilution of the reaction medium for the synthesis of MCM-41 on the hysteresis loop was studied in [39]. The authors correlated the type of hysteresis with defects present in the pores of the MCM-41 and affirmed that, for smaller proportions of water, structural defects of the type tubule-within-tubule (TWT) led to a sharp increase in the width of the loop. Therefore, it is possible that the large hysteresis loop observed in Fig. 3b for the sample MCM CV TR was due to the formation of such defects, which could have been eliminated by the increase of water proportion in the medium (sample MCM CV TR DL). To confirm this hypothesis, further analysis of the pore structure (using transmission electron microscopy, for example) would be necessary to determine the real influence of the synthesis parameters. Finally, the mesopores volume did not show notable change; both samples presented a mesoporous volume of approximately $0.68 \mathrm{~cm}^{3} \cdot \mathrm{g}^{-1}$.

Effect of TEOS added dropwise: adsorption isotherms are presented for samples synthesized with reduced stirring time, in diluted medium, without (Fig. 3c) and with (Fig. $3 \mathrm{~d})$ controlled dropping of TEOS into the solution. It was observed that the types of isotherm and hysteresis loop remained the same. However, the slope of the curves for relative pressures between 0.1 and $0.3 \mathrm{P} / \mathrm{P}_{0}$ became steeper for the sample synthesized with TEOS added dropwise; this indicated different specific surface area values. Furthermore, the results obtained show that the isotherm for the sample

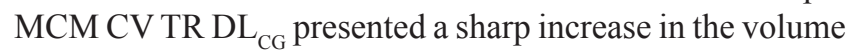
of adsorbed gas in the interval of high relative pressures. As explained before, this behavior characterizes the presence of porosity between the particles and possibly indicates agglomeration. When the clusters have a controlled feed of TEOS during the synthesis, the concentration of units of hydrolyzed silica is also controlled. Thus, one can avoid growth of the added species to a critical size and formation of a new phase, which would lead to a heterogeneous system. In addition, the addition of the remaining volume of TEOS at once would elevate the concentration of hydrolyzed monomers in the reaction medium and, consequently, increase the probability of agglomeration of the obtained particles. Therefore, TEOS added in a controlled manner promoted the control over the nuclei initially formed in the system in a way that reduced the particle size and improved the dispersion of the particles. The specific surface area decreased from 970 to $739 \mathrm{~m}^{2} \cdot \mathrm{g}^{-1}$ for the samples MCM
CV TR DL and MCM CV TR DL ${ }_{\text {CG }}$, respectively. The SEM images for those samples showed that there was a significant reduction in the particle size after controlling the dropping of TEOS. It can be concluded that this dropwise addition of TEOS was efficient to control the size of the nuclei formed during nucleation and the concentration of silica during the hydrolysis. The smaller particle size, though, resulted in more intense interparticle interactions, and the particle agglomeration led to a reduction in the specific surface area. This effect can be confirmed by the steep slope of the isotherm in regions of high relative pressure for the sample MCM CV TR DL ${ }_{\mathrm{CG}}$ (Fig. 3d). About the mesopore size, Table II shows a small increase in the mesopore average diameter after controlling the dropping of TEOS on the reaction media. The average diameters were 3.6 and $3.7 \mathrm{~nm}$ for the samples MCM CV TR DL and MCM CV TR DL ${ }_{C G}$, respectively. The mesopore volume decreased from 0.7 $\mathrm{cm}^{3} / \mathrm{g}$ (former sample) to $0.6 \mathrm{~cm}^{3} / \mathrm{g}$ (latter sample).

Effect of the addition of TEA: Figs. $3 \mathrm{~d}$ and $3 \mathrm{e}$ present the adsorption isotherms for the samples MCM CV TR DL $\mathrm{CG}_{\mathrm{CG}}$ and MCM CV TR DL TEA ${ }_{C G}$, respectively, in order to evaluate the effect of the addition of TEA to the reaction system. It is possible to observe that the volume of the adsorbed gas in the sample with TEA was more than two times higher than the sample without TEA. Also, there was a sudden increase in the curves for high relative pressures for the sample MCM CV TR DL TEA ${ }_{\mathrm{CG}}$, showing possible agglomeration of the particles. The specific surface area of the samples MCM CV TR DL $\mathrm{CG}_{\mathrm{C}}$ and MCM CV TR DL TEA ${ }_{\mathrm{CG}}$, respectively, were 739 and $919 \mathrm{~m}^{2} \cdot \mathrm{g}^{-1}$. Even though it was expected the main contribution to a more dispersed system, the addition of TEA showed the opposite effect according to the SEM images. In this case, the particles presented smaller diameters and more agglomeration. Since the sample MCM CV TR DL ${ }_{\mathrm{CG}}$ also seemed to be very agglomerated, it can be assumed that the predominant effect of the TEA in the sample was the reduction in the particle diameter instead of the increase in the dispersion. A possible explanation for this behavior is that the TEA can be also a base catalyst in the synthesis of mesoporous silica, being able to substitute the base $[2,8]$. Since in this work it was opted for keeping the base TMAOH and adding the TEA to the solution $\mathrm{A}$, it can be concluded that the TEA affected the morphology of the particles as a base catalyst and dispersion agent at the same time, but the base catalyst effect was predominant for adding to the pre-existent catalyst. The increase in the $\mathrm{pH}$ results in an increment in the silica hydrolysis rate. The preferential sites for growth during the condensation of silica monomers are, therefore, the silanol groups. In other words, the large silica monomers that are being developed stop interacting among themselves and grow by the condensation in small active nuclei, newly formed during the hydrolysis stage [40], resulting in smaller final particles. At the same time, the intensity of the van der Waals forces is stronger for smaller particles, leading to a state of higher agglomeration. The mesopore sizes can be observed in Table II: approximately 3.7 and $3.6 \mathrm{~nm}$ for the samples MCM CV TR DL ${ }_{\mathrm{CG}}$ and MCM CV TR DL TEA ${ }_{\mathrm{CG}}$, 


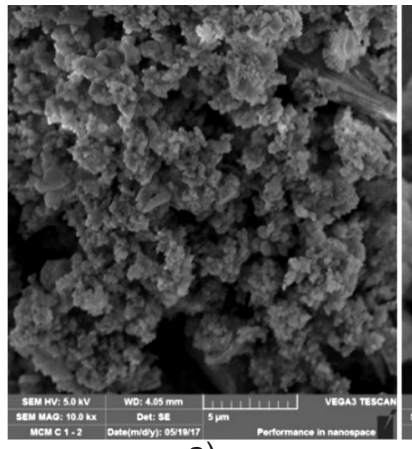

a)

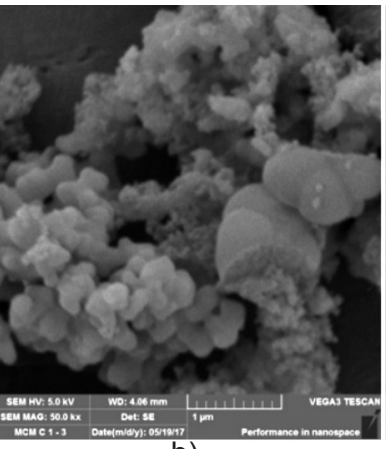

b)

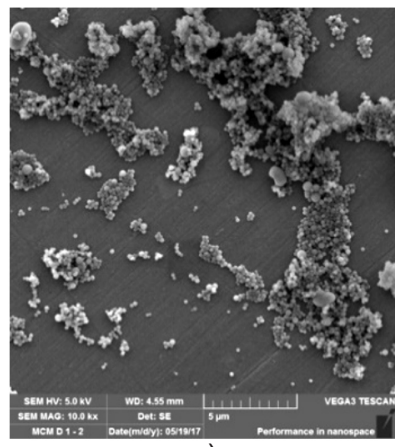

e)

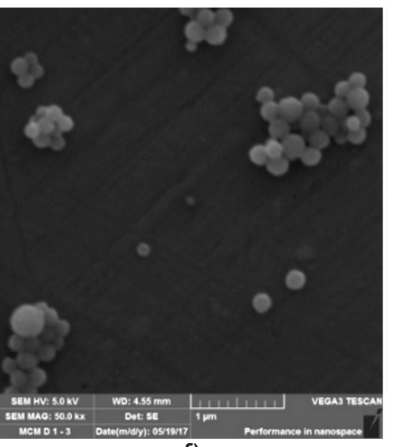

f)

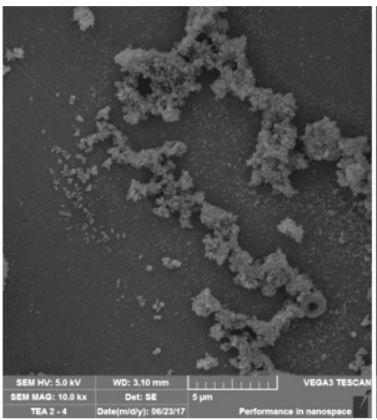

i)

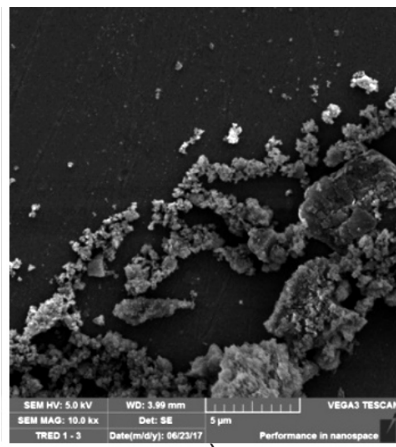

C)

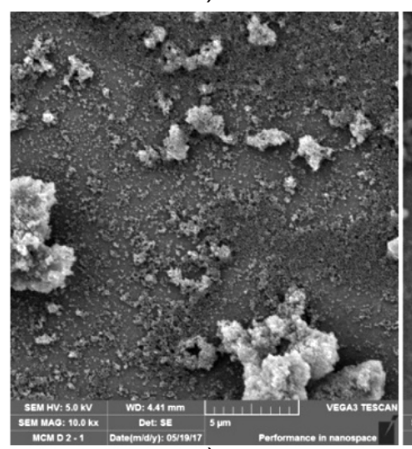

g)

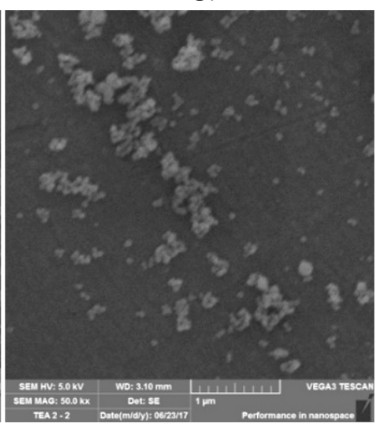

j)

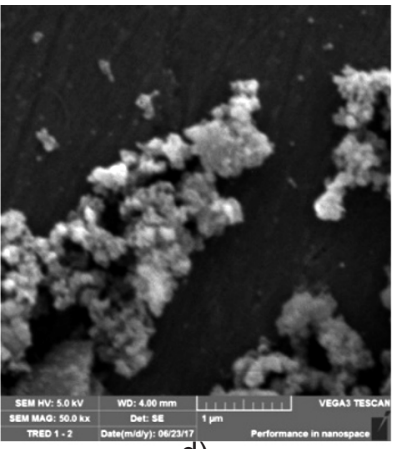

d)

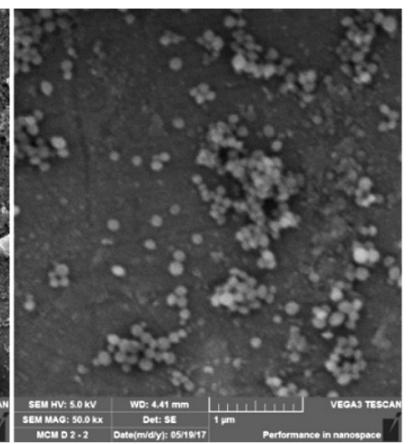

h)

Figure 4: SEM micrographs of the samples: a,b) MCM CV; c,d) MCM CV TR; e,f) MCM CV TR DL; g,h) MCM CV TR DL ; and i,j) MCM CV TR DL TEA ${ }_{\mathrm{CG}}$.

[Figura 4: Micrografias de MEV das amostras: a,b) MCM CV; c,d) MCM CV TR; e,f) MCM CV TR DL; g,h) MCM CV TR DL MCM CV TR DL TEA ${ }_{C G}$.]

respectively. The mesopores volume, however, significantly increased after the addition of TEA: approximately 1.29 $\mathrm{cm}^{3} / \mathrm{g}$ for the sample MCM CV TR DL TEA ${ }_{\mathrm{CG}}$ and 0.62 $\mathrm{cm}^{3} / \mathrm{g}$ for the sample MCM CV TR DL $\mathrm{CG}$.

\section{Scanning electron microscopy (SEM)}

The scanning electron microscopy was used with the purpose of determining the morphological characteristics of the mesoporous silica samples, as well as observing the effect of the TEOS added in a controlled manner and the efficiency of the TEA as a dispersion agent.

Effect of the reduction in the stirring time: in Figs. $4 \mathrm{a}$ and $4 \mathrm{~b}$ it is possible to see that the conventional MCM-41 (MCM CV) had its particles with an irregular shape, highly agglomerated, and with non-uniform size distribution. The agglomerates and the heterogeneity in the particle size could be a consequence of the lack of control of the dropping rate of TEOS during the synthesis, resulting in non-uniform silica precipitation. With respect to the reduction in the stirring time, the micrographs presented in Figs. 4c and $4 \mathrm{~d}$ show that the average particle size in the sample MCM CV TR decreased significantly, in the order of hundreds on nanometers, confirming that the particle growth was limited by the reaction time. In addition, there was no significant change in the shape of the mesoporous silica particles when comparing to Figs. 4a and 4b (MCM CV). The particles still seemed to be highly agglomerated and with irregular geometry.

Effect of the increase in the molar proportion of water: the micrographs presented in Figs. 4e and 4f show the effect of the dilution of the system in the shape and size of the final particles. Comparing those micrographs with the ones for the sample MCM CV TR (Figs. 4c and 4d), it can be seen that the particles were mostly spherical and very agglomerated, individually in the scale of $200 \mathrm{~nm}$. 
Effect of TEOS added dropwise: in the diluted sample, the control while dropping the remaining TEOS to the final solution promoted dispersion and reduction in the particle size. This effect can be identified when comparing the micrographs of the samples obtained in diluted medium with (Figs. 4g and 4h) and without (Figs. 4e and 4f) TEOS added dropwise. Adding smaller amounts of TEOS to the solution results in a controlled growth of the pre-existent nuclei, controlling also the nucleation of new particles [35]. Therefore, a second nucleation process from a faster polymerization rate is avoided, as well as the obtainment of a bimodal distribution of particle size. In particular, Fig. $4 \mathrm{~g}$ shows that the particles were distributed more uniformly over the surface of the sample holder. There were still agglomerates, but they were a consequence of the stronger van der Waals interactions between smaller particles.

Effect of the addition of TEA: the addition of TEA to the conventional synthesis with reduced stirring time and higher molar proportion of water (Figs. $4 i$ and $4 j$ ) resulted in nucleation of smaller particles with the same shape as the sample synthesized under the same conditions but without TEA. In addition, the expected effect of encapsulation and isolation of the particles, promoting a more disperse system, was not achieved. This was probably a consequence of the TEA acting as a dispersion agent and base catalyst at the same time, and the latter effect was predominant in the sample MCM CV TR DL TEA ${ }_{\mathrm{CG}}$.

\section{CONCLUSIONS}

The effect of the change in some parameters of the conventional methodology for the synthesis of the mesoporous silica MCM-41 in the morphology of the final particles was evaluated in this work. Particularly, it was sought a decrease in the particle diameter and an improvement in the dispersion of the system, aiming future applications in the area of drug delivery systems. The data obtained from the nitrogen adsorption analysis showed that the increase in the molar proportion of water in the reaction medium, the control over the addition of the remaining TEOS and the addition of the reactant TEA to the initial mixture of surfactant and water increased the specific surface area when compared to the conventional sample. It possibly indicated the presence of particles with a reduced diameter. The synthesis with a reduced stirring time resulted in a smaller specific surface area when compared to the conventional synthesis. In this case, it is believed that the smaller diameter was a result of the limited growth time; however, the agglomeration effect was predominant in the system. The SEM images confirmed the reduction in particle sizes after the changes in the synthesis parameters. Spherical particles with a more homogeneous size distribution were found in the samples synthesized in the diluted medium. The TEOS added dropwise, as well as the addition of TEA resulted in more dispersed particles. However, since those changes also contributed to the reduction in particle size, van der Waals interactions were intensified and the particles were still agglomerated. Therefore, it can be concluded that the reduction in stirring time, the increase in the molar proportion of water in the reaction medium, the control over the dropping of the remaining TEOS and the addition of the reactant TEA helped to obtain MCM-41 mesoporous silica particles with a smaller diameter, but totally dispersed particles were not achieved. For future work, it is suggested the search for other strategies to reduce the agglomeration of the silica particles, such as using capping and stabilizing agents and studying the stability of the dispersion through zeta potential measurements. In addition, alternative ways to remove the surfactant from the powder should be tested, such as a solvent-extraction method, since the calcination may eliminate some silanol groups, promote condensation of the particles and lead to agglomeration.

\section{REFERENCES}

[1] M. Vallet-Regí, M.M. Garcia, M. Colilla, Biomedical applications of mesoporous ceramics drug delivery, smart materials, and bone tissue engineering, CRC Press, Boca Raton (2013) 1.

[2] M. Bouchoucha, M.F. Cote, R. C.-Gaudreault, M.A. Fortin, F. Kleitz, Chem. Mater. 28, 12 (2016) 4243.

[3] M. Vallet-Regi, A. Ramila, R. Del Real, J. Perez-Pariente, Chem. Mater. 13, 2 (2001) 308.

[4] B.G. Trewyn, I.I. Slowing, S. Giri, H. Chen, V. Lin, Acc. Chem. Res. 40, 9 (2007) 846.

[5] I.I. Slowing, J.L. Vivero-Escoto, C.-W. Wu, V.S.-Y. Lin, Adv. Drug Deliv. Rev. 60, 11 (2008) 1278.

[6] M. Varache, I. Bezverkhyy, L. Saviot, F. Bouyer, F. Baras, F. Bouyer, J. Non-Cryst. Solids 408 (2015) 87.

[7] R. Singh, J.W. Lillard, Exp. Mol. Pathol. 86, 3 (2009) 215.

[8] X. Lv, L. Zhang, F. Xing, H. Lin, Microporous Mesoporous Mater. 225 (2016) 238.

[9] A. Sharma, S.V. Madhunapantula, G.P. Robertson, Expert Opin. Drug Metab. Toxicol. 8, 1 (2012) 47.

[10] Z. Yi, L.F. Dumée, C.J. Garvey, C. Feng, F. She, J.E. Rookes, S. Mudie, D.M. Cahill, L. Kong, Langmuir 31, 30 (2015) 8478.

[11] K. Yano, Y. Fukushima, J. Mater. Chem. 13 (2003) 2577.

[12] R.I. Nooney, D. Thirunavukkarasu, Y. Chen, R. Josephs, A.E. Ostafin, Chem. Mater. 14 (2002) 4721.

[13] R.P. Bagwe, L.R. Hilliard, W. Tan, Langmuir 22, 9 (2006) 4357.

[14] J.K. Bailey, M.L. Mecartney, Colloids Surf. 63, 1 (1992) 151.

[15] N.I. Vazquez, Z. Gonzalez, B. Ferrari, Y. Castro, Bol. Soc. Esp. Ceram. V. 56, 3 (2017) 139.

[16] L.F. de Oliveira, K. Bouchmella, K.A. Gonçalves, J. Bettini, J. Kobarg, M.B. Cardoso, Langmuir 32, 13 (2016) 3217.

[17] H. Giesche, J. Eur. Ceram. Soc. 14, 3 (1994) 205.

[18] K. Nozawa, H. Gailhanou, L. Raison, P. Panizza, H. Ushiki, E. Sellier, J.P. Delville, M. Delville, Langmuir 21, 
4 (2005) 1516.

[19] M.L. Saladino, E. Kraleva, S. Todorova, A. Spinella, G. Nasillo, E. Caponetti, J. Alloys Compd. 509, 35 (2011) 8798.

[20] K.S.W. Sing, D.H. Everett, R.A.W. Haul, L. Moscou, R.A. Pierotti, J. Rouquerol, T. Siemieniewska, Pure Appl. Chem. 57, 4 (1985) 603.

[21] E.P. Barrett, L.G. Joyner, P.P. Halenda, J. Amer. Chem. Soc. 73, 1 (1951) 373.

[22] C. De La Torre, I. Casanova, G. Acosta, C. Coll, M.J. Moreno, F. Albericio, E. Aznar, R. Mangues, M. Royo, F. Sancenón, R. Martínez-Máñez, Adv. Funct. Mater. 25, 5 (2015) 687.

[23] M.J. Reber, D. Brhwiler, Dalton Trans. 44, 41 (2015) 17960.

[24] M. Thommes, K. Kaneko, A. Neimark, J.P. Olivier, F. Rodriguez-Reinoso, J. Rouquerol, K. Sing, Pure Appl. Chem. 87, 9-10 (2015) 1051.

[25] P. Nguyen, D.D. Do, D. Nicholson, J. Phys. Chem. C. 115, 11 (2011) 4706.

[26] K.D. Sattler, Handbook of nanophysics: functional nanomaterials, CRC Press, Boca Raton (2010) 9-1.

[27] M. Thommes, Chem. Ing. Tech. 82, 7 (2010) 1059.

[28] E.M. Johansson, "Controlling the pore size and morphology of mesoporous silica", Thesis, Linköping Un.
(2010).

[29] R. Schmidt, M. Stöcker, E. Hansen, D. Akporiaye, O.H. Ellestad, Microporous Mater. 3, 4 (1995) 443.

[30] M. Kruk, M. Jaroniec, Chem. Mat. 13, 10 (2001) 3169.

[31] N. Jaramillo, C. Paucar, C. García, J. Mater. Sci. 49, 9 (2014) 3400.

[32] N. Thanh, N. Maclean, S. Mahiddine, Chem. Rev. 114, 15 (2014) 7610.

[33] A. Sousa, E.M.B. Sousa, J. Non-Cryst. Solids 352, 32 35 (2006) 3451.

[34] A. Sayari, Y. Yang, M. Kruk, M. Jaroniec, J. Phys. Chem. B 103, 18 (1999) 3651.

[35] J.L. Blin, B.L. Su, Langmuir 18, 13 (2002) 5303.

[36] M. Kruk, M. Jaroniec, A. Sayari, J. Phys. Chem. B 101, 4 (1997) 583

[37] S. Sakka, H. Kozuka, S.-H. Kim, in "Ultrastructure processing of advanced ceramics", J.D. Mackenzie, D.R. Ulrich (Eds.), John Wiley Sons, New York (1988) 159.

[38] T. Sugimoto, Fine particles: synthesis, characterization, and mechanisms of growth, Surfact. Sci. Series 92, Marcel Dekker, New York (2000) 1.

[39] H.-P. Lin, S.-T. Wong, C.-Y. Mou, C.-Y. Tang, J. Phys. Chem. B 104, 38 (2000) 8967.

[40] C.J. Brinker, J. Non-Cryst. Solids 100, 1 (1988) 31.

(Rec.02/07/2018, Rev. 01/10/2018, Ac. 03/11/2018) 